\title{
Association between COL1A1 gene polymorphisms and bone size in Caucasians
}

\author{
Ji-Rong Long ${ }^{1}$, Peng-Yuan Liu ${ }^{1}$, Yan Lu ${ }^{1}$, Dong-Hai Xiong ${ }^{1,2}$, Lan-Juan Zhao ${ }^{1,2}$, \\ Yuan-Yuan Zhang ${ }^{1}$, Leo Elze ${ }^{1}$, Robert R Recker ${ }^{1}$ and Hong-Wen Deng ${ }^{\star 1,1,2,3}$
}

\author{
${ }^{1}$ Osteoporosis Research Center, Creighton University, Omaha, NE 68131, USA; ${ }^{2}$ Department of Biomedical Sciences, \\ Creighton University, Omaha, NE 68131, USA; ${ }^{3}$ Laboratory of Molecular and Statistical Genetics, College of Life \\ Sciences Hunan Normal University, Changsha, Hunan 410081, PR China
}

Bone size is an important determinant of bone strength and a risk factor of osteoporotic fracture. Several studies indicate that bone size has a high heritability. Thus, a better understanding of genetic factors regulating bone size might have important clinical implications. In the present study, we examined the relationship between the collagen type I alpha 1 (COL1A1) gene and bone size at the spine, hip and wrist in a sample of 1873 subjects of Caucasian origin from 405 nuclear families. Three single-nucleotide polymorphisms (SNPs) in the COL1A1 gene were analyzed. The minor allele frequencies were 15.4, 18.8, and $1.9 \%$ for SNP1, SNP2, and SNP3, respectively. Haplotypes were reconstructed based on the family information as well as marker genotypes using the program Genehunter. We did not find evidence of population stratification, within-family association, or linkage for either single SNPs or haplotypes at any skeletal site. Suggestive evidence of total association was observed for the wrist size at SNP2 $(P=0.011)$. After adjusting age, sex, height, and weight, subjects with the $T$ allele of SNP2 had, on average, $3.05 \%$ smaller wrist size than noncarriers. When the subjects were divided into families with only female offspring and families with male offspring only, similar total associations were found at the wrist size for SNP2 with $P$-values of 0.011 and $\mathbf{0 . 0 1 0}$, respectively. In conclusion, the COL1A1 gene may have some effects on bone size variation at the wrist, but not at the spine or hip in our Caucasian nuclear families.

European Journal of Human Genetics (2004) 12, 383-388. doi:10.1038/sj.ejhg.5201152

Published online 14 January 2004

Keywords: COL1A1 gene; bone size; QTDT

\section{Introduction}

Osteoporosis is one of the most common bone and mineral disorders. It is mainly characterized by low bone mineral density (BMD), which is defined as the ratio of bone mineral content to bone size. ${ }^{1}$ Hence, bone size is a component of BMD and also a simpler phenotype than $\mathrm{BMD}$, in terms of the complexity of the influencing

*Correspondence: Dr H-W Deng, Osteoporosis Research Center, Creighton University Medical Center, 601 N. 30th St. Suite 6787, Omaha, NE 68131, USA. Tel: + 1402280 5911; Fax: + 1402280 5034;

E-mail: deng@creighton.edu

Received 2 September 2003; revised 16 November 2003; accepted 20 November 2003 factors. $^{2}$ The pathogenesis of bone fragility is heterogeneous. Low BMD is not the only important risk factor for osteoporotic fractures, but several other factors also contribute, including muscle strength, bone quality, and bone shape. ${ }^{3,4}$ Studies have suggested that bone size per se may play an important role that is independent of BMD and BMC in determining bone strength. ${ }^{5,6}$ The QTLs for BMD and bone size are different. ${ }^{7} \mathrm{~A}$ decrease in bone mass may result in a loss of bone strength, and the loss may be offset, at least in part, by an increase in bone size, tending to preserve bone strength. ${ }^{8}$ A deficit in bone size may partly account for both the increased bone fragility and the deficit in BMD relative to age-matched controls. ${ }^{6}$ 
Compared with BMD, genetic studies on bone size have been rather scarce so far, even though the genetic determination of bone size has been characterized. ${ }^{2,9}$ Generally, the heritability of bone size at the spine, hip, and wrist is over $50 \% .^{2}$ Several genomic regions and candidate genes have been shown to be linked to the variation of bone size. ${ }^{7,10}$ Collagen type $I$, the most abundant protein in bone, is composed of two alpha 1 chains and one alpha 2 chain, which are encoded by the corresponding genes, collagen type I alpha 1 (COL1A1) and collagen type I alpha 2 (COL1A2). Grant et al ${ }^{11}$ first described a $G-T$ polymorphism at the first base of a binding site for the transcription factor Sp1 in the first intron of the COL1A1 gene that was associated with low bone density and increased occurrence of osteoporotic fracture. The relevance of this polymorphism to risk of fracture was also reported to be independent of BMD and mediated through variations in bone size and geometry. ${ }^{12-14}$

In this study, we investigated three single-nucleotide polymorphisms (SNPs) in the COL1A1 gene to analyze whether they have effects on bone size variation at the lumbar spine, hip, and wrist in a large sample of Caucasian nuclear families.

\section{Materials and methods Subjects}

The study subjects came from an expanding database being created for studies to search for genes underlying the risk of osteoporosis and obesity at the Osteoporosis Research Center of Creighton University. The study was approved by the Creighton University institutional review board. All subjects were Caucasians of European origin. The exclusion criteria detailed by Deng et al ${ }^{15}$ were adopted to minimize any known potential confounding effects on the study phenotypes. A total of 1873 subjects from 405 nuclear families were recruited. All subjects signed informedconsent documents before entering the project. For each study subject, information on age, sex, medical history, and family history was acquired.

\section{Measurement}

As described in Deng et al, ${ }^{7}$ bone sizes of the lumbar spine (L1-L4), total hip, and wrist (mid-area of forearm) were measured by a dual energy X-ray absorptiometry (DXA) with a Hologic $2000+$ or 4500 scanner (Hologic Inc., Bedford, MA, USA). Bone size was reported as area measurement in units of $\mathrm{cm}^{2}$. Both scanners were calibrated daily, and long-term precision was monitored with phantoms. Measurements from the two machines were highly comparable and were well within the precision limits. ${ }^{2}$ In addition, when treating scanner type as a covariate, its effect was not significant. ${ }^{2}$ Members of the same nuclear family were measured with the same scanner.
Approximately $92 \%$ of the subjects were measured with the Hologic 4500 machine. Height and weight were measured at the same visit for bone size measurement.

\section{Genotyping}

Three SNPs in the COL1A1 gene (named SNP1-SNP3) were chosen according to the literatures ${ }^{11,16}$ and the function of the SNP, i.e., whether there is amino-acid change. DNA was extracted from whole blood using a commercial isolation kit (Gentra Systems, Minneapolis, MN, USA). The genotyping procedure for all SNPs was similar, involving polymerase chain reaction (PCR) and invader assay reaction (Third Wave Technology, Madison, WI, USA). PCR was performed in a $10 \mu \mathrm{l}$ reaction volume with 30 cycles. The sequences of the PCR primers for all three SNPs are presented in Table 2. After amplification, an invader reaction was performed in a $7.5 \mu \mathrm{l}$ reaction volume, with $3.75 \mu$ l diluted PCR product (1:20), $1.5 \mu$ l probe mix, $1.75 \mu$ l Cleavase FRET mix, and $0.5 \mu$ l Cleavase enzyme/ $\mathrm{MgCl}_{2}$ solution (Third Wave Technology). The reaction mix was first denatured at $95 \mathrm{C}$ for $5 \mathrm{~min}$, and then incubated at $63 \mathrm{C}$ for $20 \mathrm{~min}$. The genotype for every sample was called according to the ratio of the fluorescence intensity of the two dyes, which was read using a Cytofluor 4000 multiwell plate reader (Applied Biosystems, Foster City, CA, USA). After genotyping, the program PedCheck (http://watson.hgen.pitt.edu/register/soft_doc.html) was employed to verify Mendelian inheritance of all the marker alleles within each family. ${ }^{17}$

\section{Statistical analyses}

The allele frequencies of each SNP were estimated in all 1873 individuals via a maximum-likelihood method implemented in the program SOLAR (http:// www.sfbr.org/sfbr/public/software/solar). Haplotypes for all 1873 individuals were reconstructed using program Genehunter version 2.1, which is available at http:// www.hgmp.mrc.ac.uk/Registered/Option/genehunter.html. The SNPs were arranged according to their locations on the chromosome in the order of SNP1-SNP2-SNP3. The haplotype frequencies were computed in the unrelated sample, parents from 405 nuclear families. Pairwise linkage disequilibrium (LD) between the SNPs were calculated by the normalized measure, $D^{\prime}{ }^{18}$ and was examined by Fisher's exact test. ${ }^{19}$

Using the QTDT program (http://www.sph.umich.edu/ csg/abecasis/QTDT/), we tested population stratification, linkage, and association between the SNPs and the haplotypes with bone size variation at the three skeletal sites. These tests were described by Abecasis et $a l^{20}$ and Fulker et al. ${ }^{21}$ In order to prevent associations based on a few individuals with rare haplotypes that may lead to spurious 'statistical association', QTDT tests were only conducted for the three common haplotypes (shown in the 
results below). Multivariate normality of the phenotypic data is a critical assumption of the variance component approach such as those implemented in the QTDT. In the present study, all of the three phenotypes were found to deviate from normal distribution $(P<0.01)$ by performing the Anderson-Darling test, and therefore, were transformed to approach normality via the Box-Cox procedure. Both procedures were implemented in the statistical software MINITAB (Minitab Inc., State College, PA, USA). All the QTDT analyses were performed on the transformed data with age, sex, height, and weight as covariates, which generally affect bone size variation significantly. ${ }^{2,7}$

Some genes may have effects on the phenotypes either only in males or only in females, and such effects may be masked in a mixed-gender study. ${ }^{22}$ Therefore, in order to detect whether the relationship between the COL1A1 gene and bone size was modified by gender, the subjects were divided into two groups: nuclear families with daughters (female offspring families) and nuclear families with sons (male offspring families). For those families with several opposite-sex offspring, the male offspring and parents were grouped into male offspring families and the female children with the parents were used to construct female offspring families. For those parentless families, if there were at least two brothers or two sisters, they were used to construct the male offspring families or female offspring families, respectively. In this situation, phenotypes were not adjusted for sex since the parents' phenotypes were excluded in the QTDT.

In this study, the relationship of both SNPs and haplotypes with three phenotypes were tested. These tests resulted in a multiple testing problem. Therefore, a MonteCarlo permutation test ${ }^{23}$ was performed 1000 times to establish an empirical threshold, which was about 0.008 for an individual test to achieve a global significance level of 0.05 for our analyses in this study.

\section{Results \\ Characteristics of the study subjects}

In the present study, 1873 subjects from 405 nuclear families were analyzed, including 740 parents, 389 sons, and 744 daughters. The nuclear families varied in size from three to 12 individuals, with a mean of 4.62 individuals $( \pm S D=1.78) .341$ families were composed of both parents and at least one offspring. The remaining 64 families, with one or no parent, contained two or more children. There were $27.2,22.7,22.7$ and $27.4 \%$ of nuclear families with one, two, three, and more than three children, respectively, yielding a total of 1512 sib pairs. Descriptive characteristics of the children stratified by sex are presented in Table 1.

\section{Frequencies of the alleles and haplotypes}

Frequencies of the SNP alleles and haplotypes are presented in Tables 2 and 3, respectively. The minor allele frequencies were $15.4,18.8$, and $1.9 \%$ for SNP1, SNP2, and SNP3, respectively. Haplotypes were reconstructed for all 1873 individuals based on the pedigree information and marker genotypes. Seven haplotypes were inferred, while another theoretically possible haplotype, TTA, was missing in our sample. Three major haplotypes, $T G G, G G G$, and $G T G$, accounted for $97.8 \%$, and the other four rare haplotypes, TGA, TTG, GGA, and GTA, only amounted to $2.2 \%$.

Table 1 Basic characteristics of the daughters and sons

\begin{tabular}{lcc}
\hline & Daughters $(n=744)$ & Sons $(n=389)$ \\
\hline Age (year) & $37.73 \pm 10.33$ & $36.00 \pm 10.92$ \\
Height $(\mathrm{m})$ & $1.65 \pm 0.06$ & $1.80 \pm 0.07$ \\
Weight $(\mathrm{kg})$ & $70.40 \pm 16.42$ & $87.60 \pm 15.95$ \\
Spine size $\left(\mathrm{cm}^{2}\right)$ & $59.30 \pm 5.33$ & $70.59 \pm 6.56$ \\
Hip size $\left(\mathrm{cm}^{2}\right)$ & $33.29 \pm 3.14$ & $44.22 \pm 4.42$ \\
Wrist $\left(\mathrm{cm}^{2}\right)$ & $6.73 \pm 1.03$ & $9.76 \pm 1.61$ \\
\hline
\end{tabular}

Note: All data are presented as mean \pm SD of the raw phenotypic values without adjustment for covariates.

Table 2 Information and the primer sequences for the studied SNPs in the COL1A1 gene

\begin{tabular}{|c|c|c|c|c|c|c|c|}
\hline \multirow{2}{*}{ SNP } & \multirow{2}{*}{$d b S N P^{a}$ or reference } & \multirow{2}{*}{ Location } & \multirow{2}{*}{ Polymorphism ${ }^{\mathrm{b}}$} & \multirow{2}{*}{$\begin{array}{l}\text { Frequency } \\
\text { (\%) }\end{array}$} & \multicolumn{2}{|c|}{ Pair-wise $L D\left(D^{\prime}\right)^{\mathrm{d}}$} & \multirow{2}{*}{ Primers $\left(5^{\prime}-3^{\prime}\right)$} \\
\hline & & & & & SNP2 & SNP3 & \\
\hline SNP1 & Garcia-Giralt et $a l^{16}$ & $\begin{array}{l}\text { Exon } 1 \\
\text { (promoter) }\end{array}$ & G-T (-1997) & 15.4 & \multirow{3}{*}{$\begin{array}{l}0.918^{\mathrm{e}} \\
(3,543)\end{array}$} & $1.000^{\mathrm{e}}$ & F: GCACCCTGCCCTAGACCAC \\
\hline SNP2 & Grant et $a l^{11}$ & Intron 1 & G-T (Sp1) & 18.8 & & \multirow[t]{2}{*}{$\begin{array}{c}(15,770) \\
0.490 \\
(12,227)\end{array}$} & \multirow{2}{*}{$\begin{array}{l}\text { R: CCTAGTGCCAGCGACTGCA } \\
\text { F: CCAATCAGCCGCTCCCATTC } \\
\text { R: CATCGGGAGGGCAGGCTC } \\
\text { F: CTCAGCCTTCCCTGGCCAA } \\
\text { R: AGGCGGAAGTTCCATTGGCATC }\end{array}$} \\
\hline SNP3 & rs1800215 & Exon 45 & G-A (Ala897Thr) & 1.9 & & & \\
\hline
\end{tabular}

\footnotetext{
${ }^{a}$ SNP ID in the database dbSNP, www.ncbi.nlm.nih.gov/SNP.

${ }^{b}$ Bold-faced letters are the minor alleles and the information in the parenthesis is the name in the reference.

${ }^{c}$ Allele frequencies of SNPs are the frequencies of the minor alleles.

dThe numbers in the parenthesis are the pairwise physical distances in the unit of bp.

e $P<0.01$.
} 
Table 3 P-values of total association tests ${ }^{\mathrm{a}}$ between the COL1A1 gene and bone size

\begin{tabular}{|c|c|c|c|c|c|c|}
\hline Bone size & SNP1 & SNP2 & SNP3 & Haplotype $^{\mathrm{b}}$ TGG (17.1\%) & Haplotype GGG (59.9\%) & Haplotype GTG (20.8\%) \\
\hline \multicolumn{7}{|c|}{ Female offspring families } \\
\hline Wrist & 0.517 & 0.011 & 0.084 & 0.450 & 0.076 & 0.023 \\
\hline Hip & 0.823 & 0.689 & 0.143 & 0.807 & 1.000 & 0.522 \\
\hline Spine & 0.105 & 0.240 & 0.624 & 0.165 & 0.028 & 0.325 \\
\hline \multicolumn{7}{|c|}{ Male offspring families } \\
\hline Wrist & 0.888 & 0.010 & 0.340 & 0.708 & 0.127 & 0.041 \\
\hline Hip & 0.729 & 0.517 & 0.277 & 1.000 & 0.791 & 0.740 \\
\hline Spine & 0.823 & 0.065 & 0.655 & 0.777 & 0.118 & 0.106 \\
\hline \multicolumn{7}{|c|}{ Total nuclear families } \\
\hline Wrist & 0.680 & 0.011 & 0.079 & 0.512 & 0.084 & 0.026 \\
\hline Hip & 0.791 & 0.791 & 0.123 & 0.863 & 0.920 & 0.639 \\
\hline Spine & 0.262 & 0.045 & 0.663 & 0.303 & 0.022 & 0.106 \\
\hline
\end{tabular}

${ }^{\text {aT }}$ The results of tests for population stratification, within-family association and linkage were generally with $P>0.10$ and data were not shown. ${ }^{\mathrm{b}}$ The numbers in the parenthesis were the frequencies of the haplotypes. Since the frequencies of the other four haplotypes were low (they totally accounted for only $2.2 \%$ ), their effects on the phenotypes were not tested.

\section{Association and/or linkage between SNPs and haplotypes with bone size}

We did not find evidence of population stratification, within-family association and linkage for either single SNPs or haplotypes at any skeletal site. Even when the subjects were divided into female offspring families or male offspring families, we still did not get any evidence for these tests (data not shown). For the total association test, the $P$-value was 0.011 for wrist size variation at the SNP2 (Table 3). After adjusted by age, sex, height, and weight, $T$ allele carriers had an average of $3.05 \%$ smaller wrist size than noncarriers. When the sample was divided into female offspring families or male offspring families, similar results were observed between SNP2 and wrist size with $P$-values of 0.011 and 0.010 , respectively. The haplotype $G T G$ was found to be weakly associated with wrist size variation in the female offspring families, the male offspring families, and total subjects $(P=0.023,0.041$, and 0.026 , respectively). Weak evidence of total association was observed between the haplotype $G G G$ and spine size in the female offspring families and the total nuclear families with $P$-values of 0.028 and 0.022 , respectively. The other unspecified results dramatically exceeded the significance level with $P>0.10$.

\section{Discussion}

In QTDT, within-family association test is based on the allelic transmission through the nuclear family and is robust to population stratification/admixture. However, it leads to a potentially large reduction in power to detect allelic association because only the offspring from informative families are used. ${ }^{24}$ In total association test, all offspring individuals are used, further increasing the power to detect association compared to the within-family association test. However, total association test may produce misleading results in the case of population stratification/admixture. In the present study, we did not find evidence of population stratification/admixture; therefore, the more powerful test of total association was utilized.

To date, most studies of osteoporosis have focused on the possible genetic influences on BMD. ${ }^{25}$ However, BMD is not the only determinant of skeletal fragility. Bone size and geometry also determine bone mechanical strength and predict the risk of fracture independent of $\mathrm{BMD}{ }^{26,27} \mathrm{~A}$ strong genetic influence on the skeletal size has been well established, ${ }^{2,9}$ but the genes and allelic variants regulating bone size are not well defined. COL1A1 is an important candidate gene for osteoporosis, which encodes the alpha 1 chain of type I collagen, the most abundant protein of bone matrix. In this study, we have set out to investigate the relationship between the COL1A1 gene polymorphisms and bone size variation at three skeletal sites in a large sample of Caucasian nuclear families. We found that SNP2, the Sp1 polymorphism in intron 1 of COL1A1, was suggestively associated with the wrist size variation in both female and male offspring families and the total subjects as well. Although SNP2 has been reported to be associated with height, ${ }^{28}$ we did not find such an association either in the total nuclear families or the female or male offspring families. When height was excluded from the covariates, the $P$-values for the total association between SNP2 with wrist size increased from $0.011,0.010$, and 0.011 (when height was used as a covariate) to $0.059,0.042$, and 0.020 in the female offspring families, male offspring families, and total nuclear families, respectively. However, for the other skeletal sites and the other SNPs and haplotypes, the results were similar whether height was used as a covariate or not.

Functional studies have shown that SNP2 alters binding of Sp1 to its recognition site and is associated with disturbances in COL1A1 transcription, collagen protein 
production, and the biomechanical properties of bone. ${ }^{29}$ The $T$ allele of this SNP was reported to act as a risk factor to osteoporotic fracture in some studies, ${ }^{12,14}$ but not in other populations. $^{30,31}$ We also analyzed the relationship between SNP2 and BMD in this population, and the risk effect of this SNP was not observed (Long J-R, personal communication). So far, only a few studies have been conducted to address the relationship between this SNP and bone geometry or bone structure. Qureshi et $a l^{32}$ found that in British women, femoral neck-shaft angle was increased by $2^{\circ}$ in the TC/TT genotype groups compared with CC homozygotes. However, they did not find any significant difference in hip axis length or femoral neck width between different genotype groups. This result was similar to ours in that no significant association was observed between SNP2 and hip size. Our results of no association between SNP2 and spine size were consistent with the findings of Sainz et al, ${ }^{33}$ in which there was no significant difference between different genotypes in the size of the vertebrae. However, in another study, ${ }^{28}$ the $T$ allele carriers were reported to have $0.5 \mathrm{SD}$ smaller width of the lumbar vertebrae. The differences between studies in design, measurement of bone size, ethnic background, and skeletal sites make it difficult to establish concordance. ${ }^{34}$

Our suggestive significant association between SNP2 with wrist size was compatible with the results of whole genome scan performed by our group. In a sample of 53 pedigrees with 630 subjects, $^{7}$ a QTL for wrist bone size variation with a two-point $\mathrm{LOD}=3.98,75 \mathrm{cM}$ from pter, and multipoint $\mathrm{LOD}=3.01,77 \mathrm{cM}$ from pter (Figure 1 ) was observed on chromosome $17 \mathrm{q} 23$, a region quite close to $17 q 21.31-q 22$ where COL1A1 is located, $82 \mathrm{cM}$ from pter. However, one issue that should be mentioned is that there are some other candidate genes located in this region, such as T-BOX 2 ( $83 \mathrm{cM}$ from pter) and Noggin $(78 \mathrm{cM}$ from pter). In this study, we did not find the evidence of linkage for the COL1A1 gene to the wrist size. One of the possible reasons is that the linkage approach is of limited statistical power in detecting and/or confirming QTLs with currently employed sample sizes. ${ }^{35-38}$ Power calculations (http:// statgen.iop.kcl.ac.uk/gpc/) showed that we only had $12 \%$ power to detect linkage to a gene accounting for $2 \%$ of phenotypic variation with a recombination fraction of 0.05 and a false-positive error rate of 0.05 . While the power to detect association for this study was $64 \%$, assuming that a QTL explained 2\% of the phenotypic variation and the LD between the studied marker and QTL is $D^{\prime}=0.90$. However, the power reduced dramatically if the marker and QTL were not in strong LD.

There are several potential limitations in this study that should be acknowledged. First, DXA has been accepted as the most commonly used technique to assess bone phenotypes in epidemiological studies. It provides an intense, narrow beam of radiation, shortened scan time, enhanced image definition, improved precision, and a

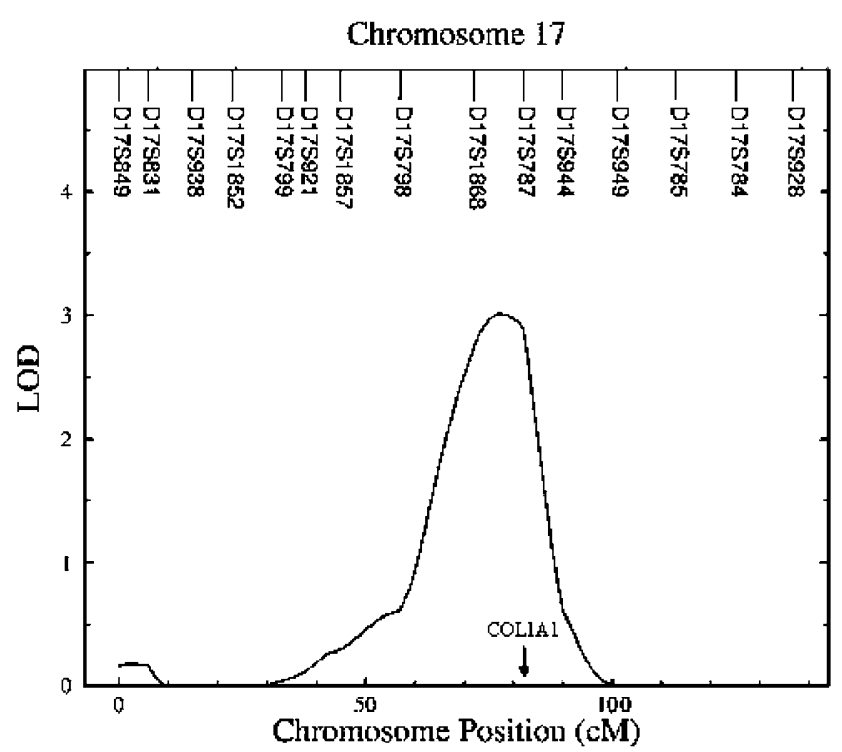

Figure 1 Maximum multipoint LOD score for wrist bone size on the chromosome 17 from Deng et $a P^{P}$ and the location of the COL1A1 gene in this region.

relative stability of calibration. ${ }^{39}$ However, it provides a two-dimensional approximation of a three-dimensional structure of bone, which can be measured by volumetric quantitative computed tomography and magnetic resonance imaging. ${ }^{40}$ However, these two methods are not readily available for large-scale studies due to the high cost, long analysis time, and a relatively high radiation exposure. Second, the children in our sample have a relatively large range of age (19-75 years); which might affect the studied phenotypes. Moreover, the total length of the COL1A1 gene was $18 \mathrm{~kb}$ and we only examined three SNPs, there being a $12.2 \mathrm{~kb}$ gap between SNP2 and SNP3. This may be the reason that we did not get evidence in haplotype analysis, although some suggestive association between SNP2 and wrist size was observed.

In summary, we have examined the association and/or linkage between the COL1A1 gene and bone size at the spine, hip, and wrist in a sample of Caucasian population. We did not find any linkage of this gene to bone size variation at any of the studied skeletal sites, while, suggestive evidence of association was observed at SNP2 for the wrist but not the spine or hip.

\section{Acknowledgements}

The study was partially supported by grants from Health Future Foundation of the USA, grants from the National Institute of Health (K01 AR02170-01, R01 GM60402-01 A1, P01 DC01813-07), grants from the State of Nebraska Cancer and Smoking Related Disease Research Program (LB595) and the State of Nebraska Tobacco Settlement Fund (LB692), and a US Department of Energy grant (DE-FG03-00ER63000/A00). 


\section{References}

1 Cummings SR, Melton LJ: Epidemiology and outcomes of osteoporotic fractures. Lancet 2002; 359: 1761-1767.

2 Deng HW, Deng XT, Conway T, Xu FH, Heaney R, Recker RR: Determination of bone size of hip, spine, and wrist in human pedigrees by genetic and lifestyle factors. J Clin Densitom 2002; 5: $45-56$.

3 Fox KM, Cummings SR, Powell-Threets K, Stone K: Family history and risk of osteoporotic fracture. Study of Osteoporotic Fractures Research Group. Osteoporos Int 1998; 8: $557-562$.

4 McCreadie BR, Goldstein SA: Biomechanics of fracture: is bone mineral density sufficient to assess risk? J Bone Miner Res 2000; 15: 2305-2308.

5 Mazess RB, Barden H, Mautalen C, Vega E: Normalization of spine densitometry. J Bone Miner Res 1994; 9: 541-548.

6 Duan Y, Parfitt A, Seeman E: Vertebral bone mass, size, and volumetric density in women with spinal fractures. J Bone Miner Res 1999; 14: 1796-1802.

7 Deng HW, Shen $\mathrm{H}, \mathrm{Xu}$ FH et al: Several genomic regions potentially containing QTLs for bone size variation were identified in a whole-genome linkage scan. Am J Med Genet 2003; 119A: 121-131.

8 Cordey J, Schneider M, Belendez C, Ziegler WJ, Rahn BA, Perren SM: Effect of bone size, not density, on the stiffness of the proximal part of normal and osteoporotic human femora. J Bone Miner Res 1992; 7 (Suppl 2): S437-S444.

9 Ferrari S, Rizzoli R, Slosman D, Bonjour JP: Familial resemblance for bone mineral mass is expressed before puberty. I Clin Endocrinol Metab 1998; 83: 358-361.

10 Koller DL, White KE, Liu G et al: Linkage of structure at the proximal femur to chromosomes 3, 7, 8, and 19. J Bone Miner Res 2003; 18: 1057-1065.

11 Grant SF, Reid DM, Blake G, Herd R, Fogelman I, Ralston SH: Reduced bone density and osteoporosis associated with a polymorphic Sp1 binding site in the collagen type I alpha 1 gene. Nat Genet 1996; 14: 203-205.

12 Uitterlinden AG, Burger H, Huang Q et al: Relation of alleles of the collagen type I alpha1 gene to bone density and the risk of osteoporotic fractures in postmenopausal women. $\mathrm{N} \mathrm{Engl} \mathrm{J} \mathrm{Med}$ 1998; 338: 1016-1021.

13 Keen RW, Woodford-Richens KL, Grant SF, Ralston SH, Lanchbury JS, Spector TD: Association of polymorphism at the type I collagen (COL1A1) locus with reduced bone mineral density, increased fracture risk, and increased collagen turnover. Arthritis Rheum 1999; 42: 285-290.

14 McGuigan FE, Armbrecht G, Smith R, Felsenberg D, Reid DM, Ralston SH: Prediction of osteoporotic fractures by bone densitometry and COLIA1 genotyping: a prospective, population-based study in men and women. Osteoporos Int 2001; 12: 91-96.

15 Deng HW, Shen H, Xu FH et al: Tests of linkage and/or association of genes for vitamin $\mathrm{D}$ receptor, osteocalcin, and parathyroid hormone with bone mineral density. J Bone Miner Res 2002; 17: 678-686.

16 Garcia-Giralt N, Nogues X, Enjuanes A et al: Two new singlenucleotide polymorphisms in the COL1A1 upstream regulatory region and their relationship to bone mineral density. $J$ Bone Miner Res 2002; 17: 384-393.

17 O'Connell JR, Weeks DE: PedCheck: a program for identification of genotype incompatibilities in linkage analysis. Am J Hum Genet 1998; 63: 259-266.

18 Lewontin RC: The interaction of selection and linkage. I. General considerations; heterotic models. Genetics 49; 49-67: 1964.

19 Chakravarti A, Buetow KH, Antonarakis SE, Waber PG, Boehm CD, Kazazian HH: Nonuniform recombination within the human beta-globin gene cluster. Am J Hum Genet 1984; 36: $1239-1258$.
20 Abecasis GR, Cardon LR, Cookson WO: A general test of association for quantitative traits in nuclear families. Am J Hum Genet 2000; 66: 279-292.

21 Fulker DW, Cherny SS, Sham PC, Hewitt JK: Combined linkage and association sib-pair analysis for quantitative traits. Am J Hum Genet 1999; 64: 259-267.

22 Duncan EL, Brown MA, Sinsheimer J et al: Suggestive linkage of the parathyroid receptor type 1 to osteoporosis. J Bone Miner Res 1999; 14: 1993-1999.

23 McIntyre LM, Martin ER, Simonsen KL, Kaplan NL: Circumventing multiple testing: a multilocus Monte Carlo approach to testing for association. Genet Epidemiol 2000; 19: $18-29$.

24 van den Oord EJ, Snieder H: Including measured genotypes in statistical models to study the interplay of multiple factors affecting complex traits. Behav Genet 2002; 32: 1-22.

25 Zmuda JM, Cauley JA, Ferrell RE: Recent progress in understanding the genetic susceptibility to osteoporosis. Genet Epidemiol 1999; 16: 356-367.

26 Peacock M, Turner CH, Liu G, Manatunga AK, Timmerman L, Johnston Jr CC, Jr: Better discrimination of hip fracture using bone density, geometry and architecture. Osteoporos Int 1995; 5: $167-173$.

27 Gilsanz V, Loro ML, Roe TF, Sayre J, Gilsanz R, Schulz EE: Vertebral size in elderly women with osteoporosis. Mechanical implications and relationship to fractures. J Clin Invest 1995; 95: 2332-2337.

28 Van Der Sluis IM, de Muinck Keizer-Schrama SM, Pols HA, Lequin MH, Krenning EP, Uitterlinden AG: Collagen I alpha1 polymorphism is associated with bone characteristics in Caucasian children and young adults. Calcif Tissue Int 2002; 71: 393-399.

29 Mann V, Hobson EE, Li B et al: A COL1A1 Sp1 binding site polymorphism predisposes to osteoporotic fracture by affecting bone density and quality. J Clin Invest 2001; 107: 899-907.

30 Liden M, Wilen B, Ljunghall S, Melhus H: Polymorphism at the Sp 1 binding site in the collagen type I alpha 1 gene does not predict bone mineral density in postmenopausal women in sweden. Calcif Tissue Int 1998; 63: 293-295.

31 Ashford RU, Luchetti M, McCloskey EV et al: Studies of bone density, quantitative ultrasound, and vertebral fractures in relation to collagen type I alpha 1 alleles in elderly women. Calcif Tissue Int 2001; 68: 348-351.

32 Qureshi AM, McGuigan FE, Seymour DG, Hutchison JD, Reid DM, Ralston SH: Association between COLIA1 Sp1 alleles and femoral neck geometry. Calcif Tissue Int 2001; 69: 67-72.

33 Sainz J, Van Tornout JM, Sayre J, Kaufman F, Gilsanz V: Association of collagen type 1 alpha1 gene polymorphism with bone density in early childhood. J Clin Endocrinol Metab 1999; 84: $853-855$.

34 Stewart TL, Ralston SH: Role of genetic factors in the pathogenesis of osteoporosis. J Endocrinol 2000; 166: 235-245.

35 Risch N, Merikangas K: The future of genetic studies of complex human diseases. Science 1996; 273: 1516-1517.

36 Allison DB: Transmission-disequilibrium tests for quantitative traits. Am J Hum Genet 1997; 60: 676-690.

37 Xiong MM, Krushkal J, Boerwinkle E: TDT statistics for mapping quantitative trait loci. Ann Hum Genet 1998; 62: 431-452.

38 Sham PC, Cherny SS, Purcell S, Hewitt JK: Power of linkage versus association analysis of quantitative traits, by use of variancecomponents models, for sibship data. Am J Hum Genet 2000; 66: $1616-1630$

39 Orwoll ES, Oviatt SK: Longitudinal precision of dual-energy X-ray absorptiometry in a multicenter study. The Nafarelin/Bone Study Group. J Bone Miner Res 1991; 6: 191-197.

40 Lang TF, Keyak JH, Heitz MW et al: Volumetric quantitative computed tomography of the proximal femur: precision and relation to bone strength. Bone 1997; 21: 101-108. 